\title{
Strategic Investments in Research in Microbiology and Immunology - Importance of Technology Infrastructure
}

\author{
Mark McIntosh, Professor and Chair, Microbiology and Immunology, \\ Director of Research Core Facilities, University of Missouri
}

\begin{abstract}
Turrent challenges to investigator driven research
The national climate for biomedical research has changed dramatically over the past 10-12 years since the last stages of the doubling of the NIH budget were completed in 2003. These changes have resulted in significant new challenges to the concept of investigator-driven basic research at medical schools and undergraduate research campuses across the nation. The biomedical research enterprise, fueled by the resources provided to the NIH and other federal research agencies during the growth period, was incentivized to recruit an expanding work force to meet the research objectives of individual investigator research grants.
\end{abstract}

The growth of individual laboratories and trainee populations was unsustainable over the long term as the federal research funds began to diminish. Federal funding in real dollars is currently $25 \%$ below the levels reached in 2003, resulting in a hyper-competitiveness for limited resources and an abundance of young investigators struggling to find researchbased academic positions or the necessary funding to launch and sustain a productive research career ${ }^{1}$. As grant application rates have risen considerably over the past 10 years, success rates have tumbled from $20-30 \%$ at the beginning of this century to the current state of $10-13 \%$. Paylines were reduced to the $6^{\text {th }}-8^{\text {th }}$ percentile for many NIH institutions, with a 3-4 percentile bump for early stage investigators (see individual NIH institutional data on the web). These historically low levels have had a depressing impact on the continued recruitment and retention of bright young scientists that are the future of our scholarly environments at research institutions. As a result, new strategies have to emerge for building research strengths and infrastructure that are interdisciplinary in nature and responsive to the changing requirements for successfully competing in the current environment.

Historically, in a medical school setting, basic science departments focused on recruiting academic expertise that centered on human clinical interests in infectious diseases, immunity and human diseases that were associated with either of these "subdisciplines". In most cases, individual faculty research interests were somewhat isolated, giving the department as a whole a breadth of perspectives that encompassed the scope of medical microbiology and immunology expertise. The teaching mission was concentrated on the one hand to providing a basic understanding of all aspects of infection and immunity, and related human physiological consequences, to an information86 
stressed medical student class through didactic lecture and case examples, and on the other to training the next generation of basic scientists to join the academy and continue the more or less siloed disciplinary environment. On an undergraduate campus, the scope of academic expertise to meet student intellectual interests expanded outside the biomedical realm to include microbial interactions with animals and plants, as well as environmental and industrial microbiology.

When a tenure track faculty position was vacated or a new position created, the recruitment strategy was to replace that expertise in a general sense - e.g. bacterial or viral pathogenesis, host immunity, etc. A search ensued by casting a broad net in the designated subdiscipline, hoping to catch the best available young scientists in that area. Most searches were targeted to the Assistant Professor level and most candidates were coming out of postdoctoral fellowships, with little or no experience with major grant applications, and thus little or no funding to initiate the transition to independence. By the time the top candidate or two was selected, interviewed and a transition package negotiated, the replacement process could be expected to take 6-12 months. That process worked well in the sense of infusing the faculty with youth, intellectual creativity and technical rejuvenation. It created many opportunities for the pipeline of young scientists being trained around the country, most supported by individual or training grants from the NIH.

Prior to 2003, the recruitment packages for faculty candidates at the Assistant Professor level were designed to give the investigator enough resources to get an independent research program off the ground and to the point of being competitive for federal grant awards. The standard calculation was three years of full salary support and $\$ 300-500 \mathrm{~K}$ to fund equipment, personnel and supplies for the transition period. This assumed no additional specialized high end instrumentation was needed or what was needed was already in place within the institution. Those estimates were defined by statistical data that suggested approximately three years from faculty appointment to the first awarded federal grant, up from one year in 1980 (2). With the first award and subsequent renewal, the institution's investment was quickly recovered. I should point out that there were also occasional targeted searches for investigators at a more senior level, with higher levels of investment required and correspondingly higher levels of expectations, including that these recruits were expected to bring significant grant resources with them along with an established and recognized research program. In other words, though there was a greater upfront investment, there was less risk in evaluating the long term potential of the recruit to maintain a productive research program.

In the period since the NIH doubling, the landscape around biomedical research has changed dramatically, especially with respect to recruitment of faculty investigators in the early stages of their careers. Young scientists are having to spend more time in prefaculty training positions because of the decline in open faculty positions. According to an $\mathrm{NIH}$ study, the average age at which $\mathrm{PhD}$ recipients are recruited into their first tenure track position is now 37 years and, 
when you couple this fact with the ever diminishing grant funding success rates, it now takes an average of 4-5 years in that position to win an initial federal grant, if at all $^{2}$. As a result, the initial investment by the institution in salary and startup must consider additional resources for the fourth and fifth years of faculty development, resources that are substantially at risk. This pushes the packages to \$700K-\$1M for an untested Assistant Professor, and higher for established investigators. Furthermore, the extended transition period for junior faculty runs headon into the tenure clocks of many institutions, which are still geared to 5-6 years. If the first award occurs near the decision year, there are no definitive data to assess long term stability for many research programs.

What about retention of not only those junior faculty with a high likelihood of success, but also of the top research faculty once their research programs have become firmly established and adequately funded? Given the fact, as will be discussed in the sections that follow, that many institutions, including MU, have begun to emphasize targeted recruitment of faculty into strategic areas of research strength and who have a strong federal funding record, what investments must be made to enhance the probability for successful strategic recruitment and reciprocally for keeping the best early stage investigators from being poached? In our collective discussions here at MU, the solutions revolve around the establishment of critical environments - a creative (innovative) and supportive environment, which includes technology enhancement, to attract the best and brightest, and an effective mentoring environment to support and retain our strongest young investigators.

1. A creative environment really depends on minimizing barriers to effective interdisciplinary research interactions, including the development of research and technology centers that promote such interactions. The era of individual investigators working with one or two students in an isolated laboratory is approaching extinction as faculty (and funding agencies) realize the effectiveness and impact of scientific collaboration to bring diverse intellectual and technical skill sets together to address significant scientific questions.

2. A supportive environment emphasizes recognition, as well as rewards (including competitive salary structures and incentives), for achieving important benchmarks in scientific discovery that bring visibility to the institution, not only in scientific citation, but in research funding as well. A supportive environment also recognizes the critical importance of investments in research infrastructure, especially with respect to cutting edge instrumentation and the technological support needed to transfer these technologies to individual/collaborative research programs.

3. An effective mentoring environment is essential in the early and sustained success of the next generation of academic scientists. A strong mentorship program will connect faculty early in their careers with established investigators who are experienced, not only 
in the relevant scientific disciplines and technologies and can provide peer review for grant applications and manuscripts, but as well in navigating the ever more difficult regulatory environment and government reporting on such issues as animal welfare, radiation safety and human subjects research. Institutionally, resources must be directed toward career development and effective teaching modalities. Faculty mentoring should also include an ongoing evaluation of how their respective research interests connect to advertised research priorities at federal agencies like the NIH.

Departmental strategies for targeted recruitment and integration into an interdisciplinary framework

We are now in a period where federal funding of basic biomedical research is not allocated in a way that affords the predictability and stability for growing research programs as it once did. To be more effective in the environment of increasing competitiveness for these critical but limited resources and to be more responsive to developing research initiatives from institutions like the NIH, public research institutions must be more creative and realistic in their approaches to investing in research scientists who fit well with interdisciplinary programmatic strengths. They must use these strengths as well to explore new funding opportunities in both the public and private sectors. This implies strategically targeted recruitments that fill important needs in currently strong research areas at the expense of the previously favored broad spectrum approach, despite the latter's evidence of success in clearly identifiable examples. Recruitments and retentions for that matter must be evaluated with integration in mind, integration with existing research strengths and integration with changing funding agency strategic plans (e.g. see NIAID Strategic Plan 2013; http://www.niaid.nih.gov/about/who-

WeAre/planningPriorities/Documents/NIAIDStrategicPlan2013.pdf).

There are clear examples from recent NIH initiatives in the Human Genome Project, the BRAIN Initiative and the Human Microbiome Project, all of which allocated impressive research resources toward establishing basic research foundations that will underpin much of the "translational" research efforts for the next decade or so.

These research initiatives, and the subsequent applicable biology, are increasingly dependent on an interdisciplinary team approach. It is imperative to build collaborative research strengths in genomics and metagenomics, bioinformatics, comparative animal models, cellular and structural biology, and drug design and development to be able to effectively compete for resources under these strategic initiatives. In parallel, there are changes in the expectations from the funding agencies for team-based research approaches, and thus there must be changes in how public research institutions respond.

1. Multi-investigator R01-type grants are gaining traction and increasing support because they bring together diverse research talents to address a research problem from a variety of technical perspectives. These grants are less expensive than the previously popular Program Project grant be- 
cause they direct all the funding resources to the science and eliminate administrative structures that are not necessary to accomplish the ultimate objectives. There are no institutional requirements that the multi-investigator team reside on the same campus; however, recruitment can be influenced by the increased likelihood of such proposals being developed when recruitment is targeted to research interactions.

2. Teamwork and complementary skill sets may be needed to overcome riskaverse scientific review panels which dominate current review processes. Many individual investigator grants can be (and are) criticized because of the limited expertise/experience of the investigator. Strategic team building eliminates this as a legitimate scientific criticism in many cases.

3. The general strategy of building interdisciplinary research teams that seek funding and publish findings together also requires that universities reevaluate current promotion and tenure policies. At present, there are significant pressures on junior faculty for "independent research" for positive tenure and promotion decisions. Within the productive interdisciplinary team, however, individual contributions are relevant to the success of the whole and can be evaluated for their respective merit.

4. As faculty become more entrepreneurial and seek new avenues for research funding from the private sector, these relationships should also be evaluated and recognized for their contributions to the academic pursuit of scientific discovery and should also be identified in faculty promotion and tenure documentation.

5. Whether in the academic setting of a university or medical school (e.g. basic/clinical integration) or in a university/industry partnership, there is an increasing emphasis on "translational applications".

For an academic research department to be effective in the research environment that is likely to dominate the national science agenda for the foreseeable future, there must be a continuous assessment of departmental research strengths relative to campus and regional intellectual and technical resources and NIH research priorities. How well do investigator research strengths integrate with campus and regional research opportunities and match with changing NIH strategic plans? From such assessments, a strategic recruitment plan should emerge that makes these integrations more effective, resulting in enhancement of opportunities to be competitive for multi-investigator research programs and a greater turnaround time for institutional return on investment.

Using the Department of Molecular Microbiology and Immunology at the MU School of Medicine as an example, the analysis of our research strengths with those of the campus as a whole and the available technical resources enable a more effective strategic plan for targeted recruitment of new faculty talent to develop. One advantage is that MU is a comprehensive campus, with multiple colleges engaging in life sciences research. As we look around campus (concentrating on areas likely to synergize with 
MMI), easily identifiable strengths include:

- Comparative medicine - with the Colleges of Medicine and Veterinary Medicine, the Division of Animal Sciences in Agriculture and three NIHfunded Animal Resource Centers (mutant mouse, rat and swine), MU has a strong cadre of research expertise in the genetic manipulation of animal models for both agricultural and medical research.

- $\quad$ lant and animal genomics - MU has long been a national leader in plant genetics and genomics and continues to invest in partnerships that enhance this strength (including the recent agreement with the Danforth Plant Science Center in St. Louis to strategically recruit four new investigators to build greater integration between the two campuses). MU has made seminal contributions to both the bovine and swine genomic projects and is a national leader in genetic manipulation of swine as animal models for human disease.

- Biological engineering and nanotechnology - Bioengineering faculty research strengths focus on nanostructured biocomposites for tissue integration, development of novel sensing mechanisms and platforms, single molecule technologies for disease biomarker detection and improved DNA sequencing, epigenetics and proteome detections, and nanoparticle development for targeted tissue delivery of molecular reagents and vaccines.
- Structural biology - Significant intellectual technical resources for crystallography and x-ray diffraction, NMR spectroscopy and mass spectrometry provide critical opportunities for determining protein structures, proteinprotein and protein-lipid interactions, and macromolecular identification.

The MMI departmental research strengths are centered on:

- Viral pathogenesis, including capsid and polymerase structure and function, antiviral therapies (small molecules and RNA aptamers), viral-cellular interactions in viral entry, replication and assembly and the use of viral vectors in gene therapy for human genetic disorders like Duchenne muscular dystrophy and spinal muscular atrophy;

- Immune response to viral and bacterial pathogens and autoimmune diseases including diabetes, multiple sclerosis, asthma, inflammatory bowel diseases;

- Bacterial genetics and pathogenesis, including membrane biology, adhesion of host cell receptors, invasion structures and functions, bacterial genetics and genetic manipulation, metagenomics of the microbiome, bacterial toxins and their molecular interactions with mammalian cellular targets and genetic diversity generating elements.

With these two comparative lists in mind, we would focus the development of a recruitment strategic plan to take advantage of the likely connections between departmental strengths, those of our comprehensive research campus and developing NIH research initiatives (along 
with entrepreneurial opportunities in the private sector). One objective of such integrated recruitment would be to create interdisciplinary centers of excellence that amplify the potential of any individual or small group of investigators to compete effectively for public or private sector resources. Integrated within this plan would also be an increasing emphasis on "translational partnerships" in the clinical sciences (human and veterinary medicine), the agricultural sciences, bio- and chemical engineering, and of course economic development. For example:

- In the area of virology and viral pathogenesis, our nationally recognized strength is in HIV/AIDS, hepatitis C and emerging viruses like Ebola and SARS/MERS. Strategic recruitment of investigators with interests in viral structures as targets for drug development, viral vaccine development and delivery and viral diagnostics will open up significant new opportunities not only for federal research programs, but for global infectious disease initiatives and pharmaceutical industry partnerships.

- In the area of microbial pathogenesis and the microbiome, we have developed a technology pipeline to interrogate the microbial metagenome within any population niche (clinical or environmental). Collaborations are rapidly developing with animal scientists interested in the ruminant microbiome and its relationship to animal health and food production, plant scientists interested in the plant rhizosphere and it role in plant nutrition and disease, human nutritionists interested in dietary caloric extraction and health/obesity, and clinical scientists interested in the relationships between the human microbiome (especially gut and vaginal) and human health/diseases. Given that NIH has already invested heavily in the foundation for studies in the human microbiome and has recently announced major new initiatives in these areas (included in Priority 3 of the recent NIAID Strategic Plan referenced above), strategic recruitment of metagenomics and informatics expertise that can collaborate with ongoing campus program development represents an exciting new opportunity.

- In the area of immunology, NIAID continues to emphasize basic innate and acquired immune mechanisms and the complex interactions between microbial pathogens and the immune system to develop and test therapeutic and vaccine strategies. In addition, new emphasis is being placed on development of the innate and acquired immune systems in relationship with the gut microbiome. Departmental research strengths in T and B cell development, immune memory and innate defenses and autoimmune diseases provide a foundation for targeted recruitment (in cooperation with Veterinary and Animal Sciences and Bioengineering) in the function of a healthy immune system, vaccine development and robust protection against bacterial and viral pathogens, and the underlying causes of diseases like diabetes, allergies and inflammation. 


\section{Technology infrastructure and de- velopment investments}

Maintaining a cutting-edge technology infrastructure is essential to creating an institutional environment where our investigators can effectively and productively meet their ongoing and developing research objectives and within which we can recruit strategic new talent. On many of our campuses, there are unique resources (like the MU Research Reactor) that bring national visibility to the institution and are a strong marketing tool for recruiting the top scientific talent. However, there is significant (and continually expanding) expense associated with highend instrumentation and the technical expertise needed for continued development of the technologies and communication of their potential to the research communities on our campuses. As a result, public institutions, in this period of diminishing state and federal revenue sources to support the necessary technological infrastructure, will benefit from innovative ideas to centralize (and not duplicate) the needed technologies and to communicate their availabilities to the local and regional scientific communities. This concept has been critically important on the MU campus and has great potential within the Missouri-Kansas region in general. I will briefly discuss how the MU campus is currently implementing a core facility infrastructure and the development of recent initiatives to communicate these technology capabilities to other regional institutions.

The real thrust to identify and consolidate our institutional technology resources began in the late 1980's with the development and implementation of a
"Core Facilities" concept. For example, MU had 3-4 electron microscopes scattered among its various colleges and not enough local resources to maintain and operate them effectively as separate instruments. Consolidating them into an Electron Microscopy Core streamlined their operational requirements and made it possible to recruit top technical talent to provide the technical expertise that campus investigators needed to justify specific experimental strategies in their grant applications. Similar consolidations and investments have been made since that time to centralize instrumentation and expertise in NMR spectroscopy, mass spectrometry (for both macromolecules and small molecules), confocal microscopy, flow cytometry, DNA and RNA genomics and transcriptomics, bioinformatics, structural biology and x-ray diffraction, and transgenic animal production. The result is a robust core facility system that is administered through the campus Office of Research (www.research.missouri.edu/division/cores). It is also important to emphasize that other technology facilities on our regional campuses are less centralized but nonetheless available for collaboration. On the MU campus, these include the VA Biomolecular Imaging Core (small animal whole body imaging), the Brain Imaging Core (with a 3T MRI), the Plant Transformation Core, the International Institute for Nano and Molecular Medicine, the Nanofabrication and Material Sciences Core and of course the MU Research Reactor.

The advantages of centralizing such technologies of course are easy to enumerate. They provide the cutting-edge technologies and state-of-the-art high end 
(and very expensive) instrumentation needed to produce competitive research proposals. They provide technical expertise on site - as investigators integrate the newest and most sensitive or quantitative technologies into their research program, they rely on the technical support and consultations with the core facility technical staff. And as importantly, they offer the services at competitive rates because they can spread the costs over many projects and investigators. Implementation of many of these technologies on a departmental or even college level would be cost prohibitive.

There are considerable challenges as well, even with a robust core facility system. (i) High end technologies are expensive from both the equipment and technical personnel perspectives. Spreading the fixed costs out to keep fee structures low and affordable requires an adequate user base. At any one institution, there may be a limited number of specific technology users (though it is absolutely essential to their research progress). According to national core facility benchmarking studies provided each year by iLab Solutions, the current rate of institutional subsidization of such technologies is $30 \%$. (ii) State-of-the-art instrumentation is evolving at a rate faster that its depreciation, making it continuously more difficult to keep pace. This is clearly apparent with genomics technologies where implementation of the latest instrument (at $\$ 500-700 \mathrm{~K}$ per instrument) is soon outdated by the introduction of a new technology. (iii) And finally, the critical technical expertise on site is increasingly difficult and more expensive to recruit and retain. Without this expertise, the technologies are available to only a select few highly trained scientists.

With the increasing costs to individual institutions of providing core technological resources, especially when the user base for any one expensive technology may be limited at a given institution, there is merit in marketing these technologies regionally. Such a strategy would benefit the institution supporting the technology by spreading costs over a stronger user base and would open new opportunities for collaboration, and it would also benefit the regional institutions by preventing the unnecessary duplication of expensive technologies. There is a strong interest from the MissouriKansas region to develop strategic partnerships that encourage and support technology sharing in this way. A consortium of institutions, including $\mathrm{MU}, \mathrm{KU}$, KU Medical Center, Kansas State, and the Kansas City Area Life Sciences Institute, with its academic, health and corporate membership, have recently undertaken a project to bring the regional technical and intellectual resources together using a web-based communications and marketing tool to illustrate the regional capabilities and academic expertise and provide its representative scientific communities a source of information for identifying needed resources. This tool, to be known as the BioInnovation Research Exchange (BRIX) is being developed in partnership with a California-based company called Assay Depot, founded by MU graduate Kevin Lustig. The tool should be ready for implementation within calendar year 2015. 


\section{References:}

1. Alberts, B., M.W. Kirschner, S. Tilghman, and H.A. Varmus. Proceedings of the National Academy of Sciences, U S A. 2014 Jul 1; 111(26):E2632-3. doi:

10.1073/pnas.1408267111. Epub 2014 Jun 11.

2. National Institutes of Health (2012) Biomedical Research Workforce Working Group Report (National Institutes of Health, Bethesda, MD)

3. NIH-NIAID Strategic Plan 2013:

http://www.niaid.nih.gov/about/whoWeAre/planningPriorities/Documents/NIAIDStrategicPlan2013.pdf
4. University of Missouri Research Core Facilities: (www.research.missouri.edu/division/cores).

5. iLab Solutions 2014 Benchmarking Study: http://www.ilabsolutions.com/benchmarking-study/

6. Kansas City Area Life Sciences Institute: http://www.kclifesciences.org/

7. Assay Depot: https://www.assaydepot.com/ 